A PROGRAM OF HADRONIC PHYSICS INVESTIGATIONS

By

E. L. Berger

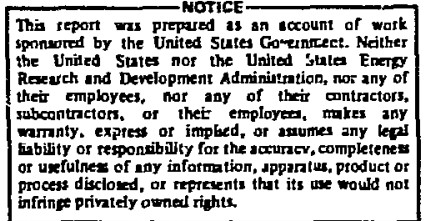


The facilities of Argonne National Laboratory are owned by the United States Government. Under the terms of a contract (W-31-109-Eng-38) between the U. S. Energy Research and Development Administration. Argonne Universities Association and The University of Chicago, the University employs the staff and operates the Laboratory in accordance with policies and programs formulated, approved and reviewed by thi Association.

\section{MEMBERS OF ARGONNE UNIVERSITIES ASSOCIATION}

The University of Arizona Carnegie-Mellon University Case Western Reserve University The University of Chicago University of Cincinnati Illinois Institute of Technology University of Illinois Indiana University Iowa State University The University of Iowa
Kansas State University The University of Kansas Loyola University Marquette University Michigan State University The University of Michigan University of Minnesota University of Missouri Northwestern University University of Notre Dame
The Ohio State University Ohio University

The Pennsylvania State University Purdue University Saint Louis University Southern Illinois University The University of Texas at Austin Washington University Wayne State University The University of Wisconsin

\section{NOTICE}

This report was prepared as an account of work sponsored by the United States Government. Neither the United States nor the United States Energy Researcl. and Development Administration, nor any of their employees, nor any of their contractors, subcontracturs, or their employees, makes any warranty, express or inplied, or assumes any legal liability or responsibility for the accuracy, completeness or usefulness of any information, apparatus, product or process disclosed, or represents that its use would not infringe privately-owned rights. Mention of commercial products, their manufacturers, or their suppliers in this publication does not imply or connote approval or disapproval of the product by Argonne National Laboratory or the U. S. Energy Research and Development Administration. 


\title{
A PRCGRAM OF HADRONIC PHYSICS INVESTIGATIONS*
}

\author{
E, L, Bergex
}

Argonne National Laboratory

The in:rease in energy provided by the Doubler offers a range of signifisant new opportunities in hadron physics investigations. In these paragraphs I discuss sore of the open quevtions which may be resolved through studies with the Doubler in its collider mode, and then with extemal beams. At the risk of being anachronistic, I concentrate on "low- $\mathrm{P}_{\mathrm{T}}$ " conventional hadron physics. The hard-core high- $\mathrm{P}_{\mathrm{T}}$ specialists have made their case vocally elsewhere at this study, as have the explorers for charm and new phenomena.

1. Doubler-Collider Physics at Small $P_{\mathrm{T}}$.

1.1 Kinematics

Ignoring the interesting $\bar{p} p$ potential, I assume that the Doubler-Collider will operate with proton beams in both rings. For reference, I adopt values $p_{1}=150$ and $500 \mathrm{GeV} / \mathrm{c}$ for the range of the momentum of the protons in bear 1 , and I take $10^{3}$ $\mathrm{GeV}$ in ring 2 . The table below lists the corresponding values of $s, \sqrt{8}$, and $\log s$.

COLLIDER

$\begin{array}{llllll}P_{1}(\mathrm{GeV} / \mathrm{c}) & P_{2}(\mathrm{GeV} / \mathrm{c} & \mathrm{s}\left(\mathrm{GeV}^{2}\right) & \mathrm{P}_{1 \mathrm{ab}}^{\text {equiv }}(\mathrm{GeV} / \mathrm{c}) & \sqrt{8}(\mathrm{jeV}) & \log 8 \\ 150 & 10^{3} & 6 \times 10^{5} & 3 \times 10^{5} & 775 . & 13.3 \\ 500 & 10^{3} & 2 \times 10^{6} & 1 \times 10^{6} & 1414 . & 14.5\end{array}$

(CERN-ISR)

31

31

$3.8 \times 10^{3} 1.9 \times 10^{3}$

62

8. 3 
Laxge $P_{T}$ processes are known to change on a scale which grows with $\sqrt{3}$, and thresholds associated with new particles and new phenomena are also supposed to be accessible in proportion to the increase of $\sqrt{\mathrm{s}}$. However, it is generally conceded that the scaling of nearly-scaling conventional $10 \mathrm{~W}-\mathrm{P}_{\mathrm{T}}$ phencsena change only on a scale measured by $(\log s)^{n}$, with $n=1$ to 3 . This theoretical yardstick may be entixely wrong, but if true it tends to make the increase in $\log s$ jovided by the Doubler/Collider look rather modest, only five to six units gain above the top of the ISR range. I belleve this conclusion is unnecessarily misleading. As I wl1 describe below, extrapolations of piesent knowledge show that the increase in log $s$ provides an important lever arm in energy and, secondly, it opens longitudinal space in the final state significantly. The increase in $\log s$ should be viewed in terms of "correlation lengths" in rap.dity. 1 From studies of rapidity correlations, this unit has been determined to be roughly 2 . Thus, the collider provides a gain of from 2 to 3 correlation lengths in longitudinal phase space. Since the fragmentation ends of the rapidity distribution each account for 1 or 1.5 of these characteristic lengths, independently of s we see that the central region of phase space is enlarged from about 2 at the ISR to 5 correlation lengths. This increase should allow clean sepazation and study of the vartous hadronic reaction mechaniams ${ }^{2}$ wich have been identified in studies at Fermilab and at the ISR. 
Some of what I include here is a modification of paragraphs I prepared earlier for the POPAE proposal. The results of the firat experiments at Fermilab and the CERN ISR quickly changed our ldeas about hadronic physics and simple extrapolations to asymptopia. The rise with energy of the proton-proton total cross section, evidence for diffraction minima in the elastic and exclusive inelastic differencial cross sections, the relatively large value of the inelastic diffractive cross section, the unexpected cojlous production of hadrons at high transverse momentum, the openirs of central ploateau in the rapidity spectrum of produced pions, and the short-range nature of inclusive correlaclons have all had important impact. While many important questions have been answered, new ones have been raised.

\subsection{Total Cross Seccions}

At Ferailab and the ISR, the total proton-proton cross section tises, the forward elastic diffraction peaks shrinks, and the real part of the forward elastic amplitude becomes positive. ${ }^{2}$ Wil this behavior continue indefinicely, is it rather the threshold onset of the asymptotic dowain with properties still unknown, or is it merely a local fluctuation? Theoretical apeculations include all of these possibilities. Data are shown in Fig.1. If we regard the present Fermilab/ISR data as suggesting the beginning of an asymptotic domain, the lever arm provided by the Collider is long probe indeed.

If the growth of the total cross section is parametrized as $a_{t}=a+b \ln ^{2} s$, the coefficient $b$ is found to be about 
$0.5 \mathrm{mb}$, two orders of magnitude below the Froissart bound, Using this formula, we expect $a_{t} \vee 80 \mathrm{mb}$ in the Collider energy range, roughly twice the mean ISR value as shown In Fig.1, A simple logarithmic parametrization also fits the ISR data: $\sigma_{t}=(25.5+2.14 \mathrm{lns}) \mathrm{mb}$. Extrapolating, we find $\sigma_{t}=55 \mathrm{mb}$, roughly one and one-half times the ISR value. Other possibilities include a power-like rise $\left(s^{0.04}\right)$, with the small power being a measure of the distance above unity of the bare Pomeron in the Gribov-Reggeon calculus, or, even a leveling off to some new constant value.

The integrated elastic cross section, $\sigma_{e l}$, and the integrated inclusive diffractive cross section, $\sigma_{\text {diffe }}$, both of order $8 \mathrm{mb}$ at ISR energies, also grow with energy in the ISR range, ${ }^{3}$ (ifg.2). Their rate of growth is consistent with being the same as that of $\sigma_{t}$, but the energy range is too narrow to $p$ in down the issue. An important prediction of mos: models is the energy dependence of the ratios $\sigma_{e l} / \sigma_{t}$ and $\sigma_{\mathrm{diff}} / \sigma_{t}$. In a geometrical scaling framework, the ratio $\sigma_{e} / \sigma_{t}$ remains constant, and the common rate of growth of $\sigma_{t}$ and $\sigma_{e l}$ measures the rate of growth of the proton's radius.

\subsection{Elastic Differential Cross Section}

Three kinematic regions of interest may be identified in the study of the elastic differential cross section. At very small $|t|$, one observes Coulomb-nuclear interference effects, from whick one may extract the phase of the hadronic amplitude. At somewhat larger $|t|$ values, $0.1<|t|<1.0 \mathrm{GeV}^{2}$, 
effects characteristic of the proton's profile in impact parameter space are studied. At still larger $|t|$ values, one probes hadronic matter at very small distances ( $<0,2$ fermi).

The elastic data presently show a shrinking forward peak. ${ }^{4}$ The logarithmic slope parameter $b=\left[\frac{d}{d t} \ln \frac{d \sigma}{d t}\right]_{t=0}$ increases as in $s$ in the ISR range, (Fig.3). Extrapolating to Collider energies, we expect $b \sim 17 \mathrm{GeV}^{-2}$. An important theoretical issue is whether the logarithmic shrinkage continues, becomes quadratic in In $s$ or stops.

A break in the slope of $\mathrm{do} / \mathrm{dt}$ near $t=-1.2 \mathrm{GeV}^{2}$ in the 10 to $20 \mathrm{GeV}$ energy range develops into a clear diffractivelike dip near $t=-1.4 \mathrm{GeV}^{2}$ at Fermilab and ISR energies, 5 as shown in Fig.4. Insight will be gained from studies of the $s$ dependence of the dip position. In a geometrical scaling model, ${ }^{6}$ the dip moves as $\sigma_{t}^{-1}$ and is therefore expected near $|t|=1 \mathrm{GeV}^{2}$ at Collider energies. Beyond the dip, a secondary maximum is observed, and for $|t|>2 \mathrm{GeV}^{2}$, a very shallow slope is measured with $\mathrm{d \sigma} / \mathrm{dt} \sim 3 \times 10^{-3} \exp (1.81 \mathrm{t})\left(\mathrm{mb} / \mathrm{Gev}^{2}\right)$ at $\sqrt{8}=53 \mathrm{GeV}$. At these large values of $|t|$, the data show no variation with energy in the ISR range. 5 This plus the shallow slope suggest that the hard scattering limit may already have been reached. If so, the same distribution should be observed at Collider energies. On the other hand, using the geometricalscaling hypothesis, we expect the large $|t|$ cross section to shrink. At $\sqrt{8}=2000 \mathrm{GeV}$, it is predicted to behave as

$$
\frac{d \sigma}{d t}=5.5 \times 10^{-3} \exp [2.5 t] \mathrm{mb} / \mathrm{GeV}^{2} .
$$


Measurements out to $|t| \sim 8 \mathrm{GeV}^{2}$ should be well within the range of possibilities at Collider energies. After sufficient shrinkage, will the large $|t|$ cross section reveal a second diffractive dip?

In experiments with external beams, are large $|t|$ dips observed in the $\pi p, K p, \Lambda p, \ldots$ elastic differential crosssections at high energies? Where or why not?

\subsection{Inelastic Diffraction}

Important diffractive effects are observed in inelastic processes, in both inclusive and exclusive processes. In the exclusive reaction $p p+\left(n_{\pi^{+}}\right)_{p}$ at the ISR, and in $n p+\left(p^{-}\right)_{p}$ at Fermilab, a dip is observect in the production differential cross section near $|t|=0.2 \mathrm{GeV}^{2}$. The dip position moves to larger $|t|$ values as the excitation mass increases. While the full meaning of these data is not yet clear, one important deduction is that the elastic and inelastic diffractive processes are very different. 8,9

A central question in the further study of diffraction is whether the Pomeron, as a factorizable t-channel singularity. may be exchanged twice. If so, one expects a double-Pomeron amplitude leading to particle production in the centrel region of rapidity space, At the highest ISR energies, the available rapidity interval is barely large enough to allow kinematic separation of the conjectured double-Pomeron signal from the large background of single diffractive excitation. In doubling the range of avallable rapidity, the Collider may provide an 
unambiguous test of thils important concept, while simultaneously allowing the subenergy of the Pomeron-Pomeron system to approach the present overall ISR energy.

The inclusive inelastic process $p p \rightarrow p X$ at sma11 $|t|$ and large $x(>0.90)$ is describea theoretically by the triple-Regge formalism. At Collider energies, masses $M_{x}$ of more than $300 \mathrm{GeV}$ are expected to be excited diffractively. One important deduction from Fermilab and ISk data is that the triple-Pomeron term appears not to vanish as $|t|+0$. The validity of this conclusion could be subject to a very stringent test at Collider energies, and the detailed dependence of the triple Regge amplitudes on $M_{x}$ will be established.

\subsection{Multiparticle Production}

The nondiffractive production of particles at small transverse momentum accounts for the bulk of the lnelastic cross section ( $2 / 3)$. Changes in the character of this dominant mechanism may be substantial over an energy range in which the total cross section could double.

In proton-proton collisions with $\sqrt{\mathrm{s}}>10 \mathrm{GeV}$, the rate of growth of the mean multiplicity of charged hadrons may be parametrized $^{10}$ as $<\mathrm{n}_{\mathrm{ch}}>\sim-3.19+1.84$ ln s. At $\sqrt{8}=1000 \mathrm{GeV}$, this expression suggests an average of 22 charged hadrons per inelastic coll ision. Higher values are predicted by parametrizations including terms such as $(\ln s)^{2}$ or $s^{n}$, as shown in Fig.6.

As predicted by models based on short-range-order concepts, and supported by data from Fexmilab and the ISR, it is generally believed that the inclusive yield of these hadrons will be 
characterized by a plateau in rapidity space. Multiperipheral and other models, such as the Mueller-Regze approach, which are dominated by short-range order, require that the magnitude of the inclusive corcelations between hadrons should decrease rapidly as the rapidity spacing between the hadro., is increased. While this expectation is consistent with the ISR data, other requirements of pure short-range order models fail seriously. The height $h(s)$ of the rapidity plateau is predicted to reach a constant limit as energy Increases, whereas the ISR data ${ }^{11}$ in Fig. 7 show a rate of increase of the plateau height for pions as much as two or three times that of $\sigma_{t}$. Pure short-range order is also rulec out by the observed growth of $\sigma_{t}$ itself. As of yet, no self-corsistent hadron theory explains the rise of $\sigma_{t}$ and the behavior of the inclusive spectra. Promising models exist, based on the Reggeon calculus, ${ }^{12}$ for example. Long-range cor:elations are expected to play a significant role in our eventual understanding. Isolating the long from the shortrange components of the correlations in various experimental distributions is now impossible even at the highest ISR energy because the phase space is too restricted. The full rapidity interval provided by the Collicier may make it possible both to isolate and to study the properties of long-range inclusive correlations. For further discussion of correlations, consult the article "Some Issues in Hadron Physics" elsewhere in these proceedings. 


\section{Extemal Beams}

The doubler both extends the momantum range and provides an important increase in the intensity of external secondary beams. In this section I discuss a tew interesting external beam experiments, again, al ys in the small- $\mathrm{p}_{\mathrm{T}}$ domain. I divide the remarks into two categories. first, reactions for which the cross-section is presumed to be roughly energy independent ("Pomeron physics"), and in Sec. 3 exchange processes having cross-sections which decrease as a power of the incident momentum. Obviously some of the remarks made in Sec. 1 for pp collisions apply also for external secondary beam physics. The list is hardly exhaustive.

\subsection{Total and Elastic Cross-Sections}

Section 1.1 applies for all beams: $\pi^{ \pm}, k^{ \pm}, \bar{p}, \bar{\Lambda}$, charm, . . The $k^{+} p$ results at high energy are interesting in that the "asymprotic" rise with energy appears to set in at a lower energy for $k^{+} p(\sim 30 \mathrm{GeV} / c)$ than for protons $(270 \mathrm{GeV} / c)$. If the present rise with energy of the total cross-sections should be a transient threshold phenomenon, perhaps a new flat behavior will appear first in the $k^{+} p$ results.

Does the $\bar{p}$ p total cross-section always stay above the pp value?

What is the energy dependence of the $\pi^{-} \pi^{-}$total crosssection? Does its asymptotic rise begin as low as the first prominent inelastic threshold ( $\sim \mathrm{GeV} / \mathrm{c}$ )?

The structure observed at various $|t|$ values in elastic differential cross-sections is not understood. Experiments with 
different beams will allow us at least to sort out the $s$ and $t$ channel quantumi numbers of the structure, and its $s$ dependence, Because there are fewer spin amplitudes in meson-baryon scattexing (and fewer quarks in the beam) meson-baryon processes may be easier to understand.

\section{$\underline{2.2 \text { Inclusive Processes }}$}

In "Some Issues in Hadron Physics" elsewhere in these proceedings, I comment on the importance of two particle inclusive correlations. I will not repeat those remarks here.

Triple-Regge processes such as $h_{1} p+h_{2} X$ have a phenomenology of their own and will especially benefit from an increase of the incident energy. ${ }^{13}$ The applicability of the model is restricted to large $M_{x}^{2}$ and large $s / M_{x}^{2}$. Requiring $M_{x}^{2}>10 \mathrm{GeV}^{2}$ and $s / M_{x}^{2}>10$, we see that $s$ must be $>100 \mathrm{GeV}^{2}$, or $p_{1 a b}>50 \mathrm{GeV} / \mathrm{c}$. It will be interesting to sort out the different triple-Regge terms $^{23}$ once a good set of data is available. Valuable processes include $\pi^{ \pm} \mathrm{p} \rightarrow \pi^{ \pm} \mathrm{X}, \mathrm{K}^{ \pm} \mathrm{p} \rightarrow \mathrm{K}^{ \pm} \mathrm{X}, \pi^{-} \mathrm{p} \rightarrow \pi^{0} \mathrm{X}$, as well as the toooften forgotten inclusive resonance production processes, e.g. $h p \rightarrow\left(\rho, \phi, \eta, \eta^{*}\right) x$. The impact of the larger than expected measured values of the inclusive $\mathrm{e}^{-} / \pi^{-}$, and $\mu^{-} / \pi^{-}$ratios as a function of $\mathrm{p}_{\mathrm{T}}$ would certainly be more evident if we had a more complete set of data on the vector meson and of the $\eta$ and $\eta^{\text {* }}$ inclusive yields as a function of $\mathrm{P}_{\mathrm{T}}$. Some of the "new physics" may not be so novel. 


\subsection{Is the pomeron a trichaniel exchiange?}

The elastic scattering amplitude can be parametrized as if it were. Its contribution to the elastic amplitude is roughly exponential and ractorization seems to be satisfied near $t=0$. However, in exclusive inelastic processes, such as $\mathrm{np} \rightarrow\left(\mathrm{p}^{-}\right) \mathrm{p}$ and $\mathrm{pp} \rightarrow\left(\mathrm{n}^{+}\right) \mathrm{p}$, a marked dip is observed near $|t|=0.2(\mathrm{GeV} / \mathrm{c})^{2}$ in $\mathrm{d}^{2} \alpha / \mathrm{dt} \mathrm{dM}^{2}$ for values of $M$ near threshold. 7 The Pomeron's $t$ dependence is therefore very different in elastic and in inelastic processes (central. versus peripheral when restated in impact parameter language ${ }^{8,9}$ ). Further progress in understanding this difference might come from good measurements of the polarization in $\mathrm{pp}+\left(\mathrm{n} \pi^{+}\right) \mathrm{p}$. I have in mind the kinematic configuration in which a polarized target is used and the $\left(n \pi^{+}\right)$is fast in the laboratory (beam dissociation). Data on proton target dissociation from a polarized proton target would be useful for establishing the spins and parities of the $N^{*}$ resonances which are apparently produced diffractively. ${ }^{7}$

Recently a SLAC group ${ }^{14}$ completed a very high statistics experiment on $\mathrm{K}^{ \pm} \mathrm{p} \rightarrow\left(\mathrm{K}^{ \pm} \pi^{+} \pi^{-}\right) \mathrm{p}$. They identify one and perhaps two resonances near $1.3 \mathrm{GeV}$ in the $\left(\mathrm{K}^{ \pm} \pi^{+} \pi^{-}\right)$system, which appear to be produced diffractively. A similar analysis should be carried out at much high energy, e.g. $200 \mathrm{GeV} / \mathrm{c}$. Does the $\mathrm{K} \rho\left(\mathrm{K}^{*} \pi\right)$ subsystem continue to be produced with $s(t)$ channel helicity conservation at much increased incident momentum?

Assuming that the Pomeron is a $t$-channel exchange and not some purely direct-channel shadow effect, we can draw diagrams 
In which it is exchanged twice, as in Fig, $5(\mathrm{a})$, In the double Pomeron process sketched in Fig, 5(a), the central vertex may represent an inclusive configuration (anything, sumed over) or an exclusive state such as $\pi^{+} \pi^{-}$or $\mathrm{K}^{+} \mathrm{K}^{-}$with $I=0$, It will be of great value theoretically ${ }^{15}$ to establish whether a double Pomeron signal is present, and the nature of the distributions in $t$ associated with the Pomeron exchanges. A practical advantage of external beams is that the momentum transfers can be more readily restricted to be very small.

\subsection{Does the Pomeron Flip Spin?}

Data from Serpukhov on the energy dependence of $\pi p+A_{2} p$ suggest that it does. 16 These measurements should be extended to much higher energies. Does the Pomeron contribute in a spin-flip mode also to $\mathrm{K}^{ \pm} \mathrm{p}+\mathrm{K}_{890}^{*} \mathrm{p}$ ?

If there is a spin-flip coupling of the Pomeron to the nucleons in $\pi^{ \pm} \mathrm{p}+\pi^{ \pm} \mathrm{p}$, we may expect interesting polarization effects at high energy $\left(p_{1 a b}>100 \mathrm{GeV} / \mathrm{c}\right)$. At low energy, the famous and well understood mirror symmetry rule holds whereby $P\left(\pi^{+} p\right)=-P\left(\pi^{-} p\right)$, and $P \propto 1 / \sqrt{s}$. If a Pomeron-Pomeron interference term dominates the polarization at high energy, we should find an energy independent polarization parameter, with $P\left(\pi^{+} p\right)=+P\left(\pi^{-} p\right)$.

3. Exchange Processes and Spectroscopy .

Many reactions of substantial dynamical interest have crosssections which axe expected to fall as an inverse power of laboratory momentum. Existing data on these processes from ANL, BNL and the CERN PS are usually limited to 1 ab momenta $\approx 20 \mathrm{GeV} / \mathrm{c}$, although scme higher energy results are available from Serpukhov. 
The range of momentum values of relevance at Fermilab is from 10 to roughly $200 \mathrm{GeV} / \mathrm{c}$,

A few showcase charge-exchange processes are 1lsted below, along with their $t$ channel Regge exchanges.
Reactions
Exchanges
1. $\pi-p \rightarrow \pi^{\circ} n$
$\rho$
2. $\pi p \rightarrow n n$
$\mathrm{A}_{2}$
3. $\quad \mathrm{K}^{-} \mathrm{p} \rightarrow \overline{\mathrm{K}}^{\circ} \mathrm{n}$
$\rho-\mathrm{A}_{2}$
4. $\mathrm{K}^{+} \mathrm{n} \rightarrow \mathrm{K}^{\circ} \mathrm{p}$
$\rho+A_{2}$

Data from the Caltech Fermilab experiment ${ }^{17}$ on $\pi^{-} \mathrm{p}+\pi^{0} \mathrm{n}$ suggest that simple Regge pole exchange model ideas are valid to an impressive degree throughout the Fermilab energy range. It would be useful to complement these data with a detailed study of the reactions 3 and 4 , above. These are related by line-reversal in the $t$-channel and should have identical differential cross-sections if duality/exchange-degeneracy ideas are valid.

The hypercharge exchange processes

5. $\pi p \rightarrow k \Lambda$

6. $R p \rightarrow \pi \Lambda$

are mediated by $\left(\mathrm{K}^{*}, \mathrm{~K}^{i * k}\right)$ sxchange. A diligent investigation of these processes, including a polarized target and determination of $\Lambda$ polarization would yield directly the two complex spin amplitudes. 18

Regge exchange phenomenology appears inconclusive to many, but sight should not be lost of its many qualitative successes. 19 
It is defensible to attribute at least some of lts apparent failures to over-exploitation in an age in which data were not available in the appropriately high range of energies, With $p_{1 a b}>20 \mathrm{GeV} / \mathrm{c}$ in the Meson Area at Fermilab, the model may finally be tested properly.

Conventional spectroscopy may yield many rewards (even when viewed as a mere by product of the search for charmed hadrons). The discovery ${ }^{20}$ of the $h$ meson at Serpukhov was a triumph of painstaking investigation. It presumably has spin-parity $\mathrm{J}^{\mathrm{P}}=4^{+}$, and lies on the $(\rho, f, g, h \ldots)$ Regge trajectory. Nothing is known experimentally about its strange (and otherwise) partners in the $4^{+}$SU(3) nonet. The $g$ meson $\left(J^{P}=3^{-}\right)$is also a relatively lonely member of an as yet incomplete multiplet. I will refrain from mentioning the sordid state of the $J^{P}=1^{++}$ and $1^{+-}$multiplets. Neutral decay modes often go undetected for technical reasons. Narrow high mass states with primarily elestromagnetic decays modes $\left(+r,+e^{+} e^{-}, \ldots\right)$ may await discovery. These states are of interest in their own right and may explain the large values of the inclusive $\mathrm{e}^{-} / \pi^{-}, \mu^{-} / \pi^{-}$, and $Y / \pi$ ratios as a function of $\mathrm{p}_{\mathrm{T}}$.

Finally, double-peripheral processes deserve some emphasis. A typical example is sketched in Fig.5(c). The diagram contributes to the region of phase space defined by small

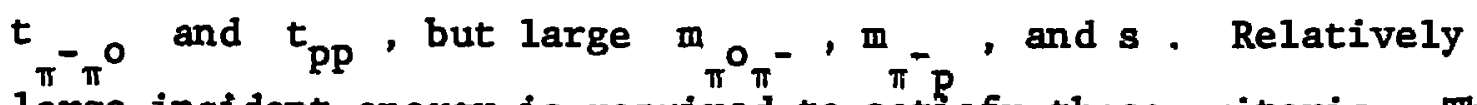
large incident energy is required to satisfy these criteria. The data are Important for a precise verification that exchange ideas 
can be extended beyond $2+2$ processes, They will give us a valuable measure of the nature of the two-Reggeon single-particle vertex, which is a butlding block in many a theoretical edifice.

\section{REFERENCES}

1. E. L. Berger, in Laws of Hadronic Matter, 1973 Erice Int. School of Subnuclear Physics, Editor, A. Zichichi (Academic Press, N.Y.) p. 557.

2. A. N. Diddens, Plenary Session Report on Low Multiplicity Reactions, XVII Int. Conf. on High Energy Physics, London,1974.

3. CERN-Holland-Iancaster-Manchester collaboration, M. G. Albrow et a1., Nuc1. Phys. B108, 1 (1976).

4. U. Amaldi, Rapporteur's report, II Aix-en-Provence Int. Conf. on Elementary Particles, 1973.

5. CERN-Hamburg-Orsay-Vienna Collaboration, H. DeKerret et al., Phys. Letters 62B, 363 (1976).

6. V. Barger, Plenary Session Report on Reaction Mechanisms at High Energy, XVII Int. Conf. on High Energy Physics, London, 1974.

7. CERN-Hamburg-Orsay-Vienna ISR Collaboration, H. DeKerret et al., CERN reports 1976 submitted to Phys. Letters, J. Biel et al., Phys. Rev. Letters 36, 504 (1976).

8. E. L. Berger and P. Pirila, Phys. Rev. D12, 3448 (1975).

9. H. I. Mtettinen, Diffractive Processes and the Triple Pomeron, Plenary Session Report, Palermo Conference, 1975.

10. E. Albini, P. Capiluppi, G. Giacomelif, and A. Rossi, Bologna preprint, Nov. 1975, submitted to Nuovo Cimento. 
11. British-Scandinavian ISR Collaboration, Brian Duff, private communication.

12. V. N, Gribov and A. A, Migdal, Sov. J, Nucl, Phys, ㅇ, 583, 703 (1969).

13. R. D. Fteld and G. C. Fox, Nucl, Phys, B80, 367 (1974).

14. G. W. Brandenburg et al., Phys, Rev. Letters $\underline{36}, 703$ and 706 (1976).

15. D. M. Chew, Nucl. Phys. B82, 422 (1974).

16. Yu. M. Antipov et al., Nucl. Phys. B63, 153 (1973).

17. A. V. Barnes et a1., Phys. Rev. Letters 37, 76 (1976).

18. E. L. Berger and G. C. Fox, Phys. Rev. Letters 25, 1783 (1970).

19. G. C. Fox and C. Quigg, Ann, Rev. of Nucl. Sci. 23, 219 (1973).

20. W. D. Apel et al., Phys. Lett. 57B, 398 (1975). 


\section{FIGURE CAPTIONS}

1. Data on the PP total cross section and two representative phenomenological extrapolations into the Colilider energy range.

2. Inclusive diffractive cross-section $\sigma_{\text {diff }}$ defined as the integral for $x \geq 0.9$ of the proton inclusive yield from $p P+p X$. ISR data (Ref.3) are shown with two sample extrapolations.

3. Energy dependence of the logarithmic slope of the forward elastic pp differential cross-section.

4. Data from the CHOV-ISR collaboration (Ref.5) on the large $|t|$ behavior of the pp elastic differential cross-section.

5. (a) Double-Pomeron exchange diagram.

(b) A triple-Regge process; $h_{1}$ and $h_{2}$ are hadrons. (c) Double-peripheral exchange diagram for $\pi^{-} p+\pi^{0} \pi^{-} p$.

6. The average multiplicity of charged hadrons as a function of energy, along with two possible extrapolations, from Ref. 10.

7. The height of the inclusive plateau for $\pi^{-}$production in the central region of rapidity space $\left(\mathrm{y}_{\mathrm{cm}}=0\right)$. The straight line is $-2.73+6.4 \mathrm{log} \sqrt{\mathrm{s}}$. From Ref. 11 . 


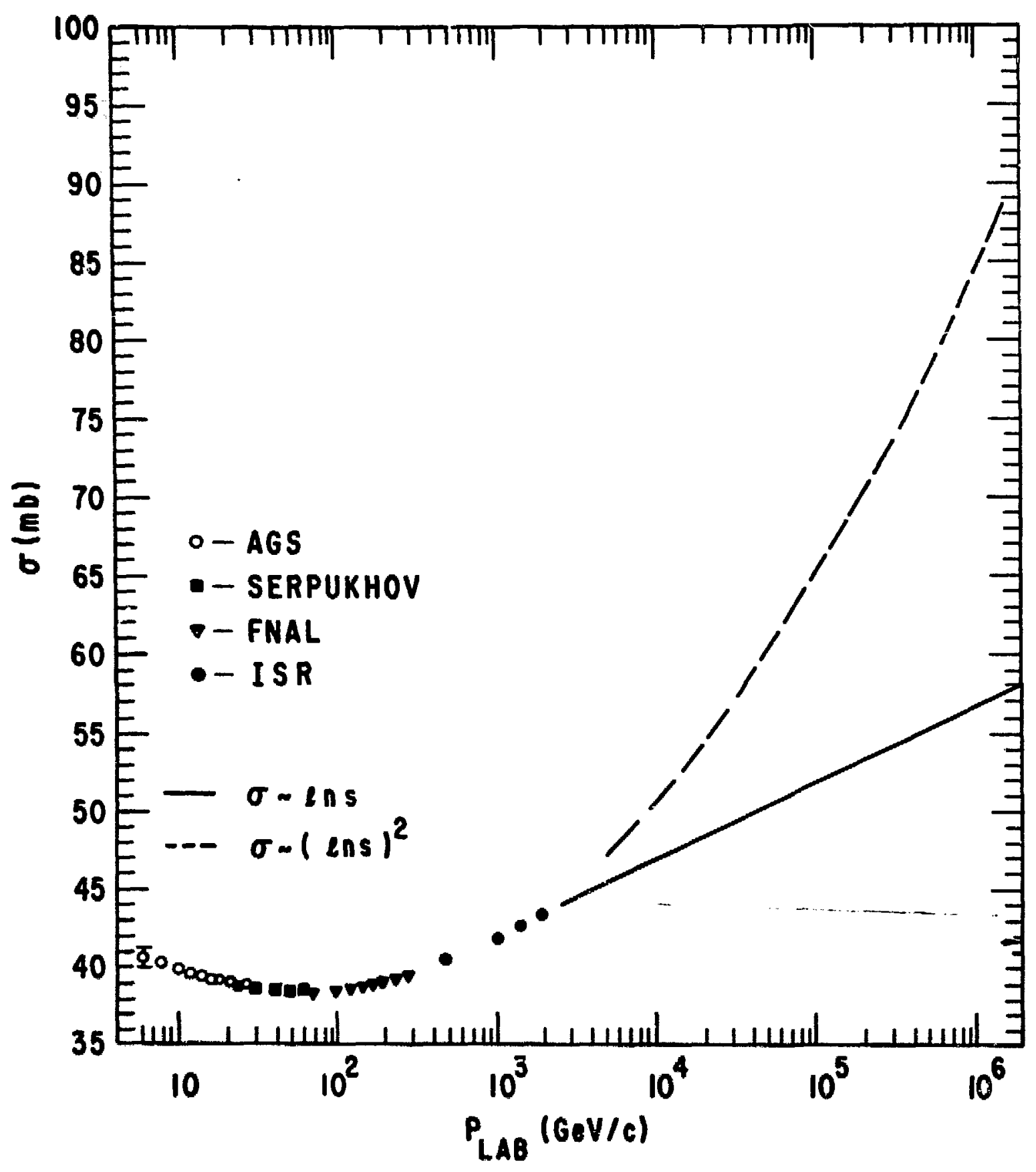

Fig. 1 


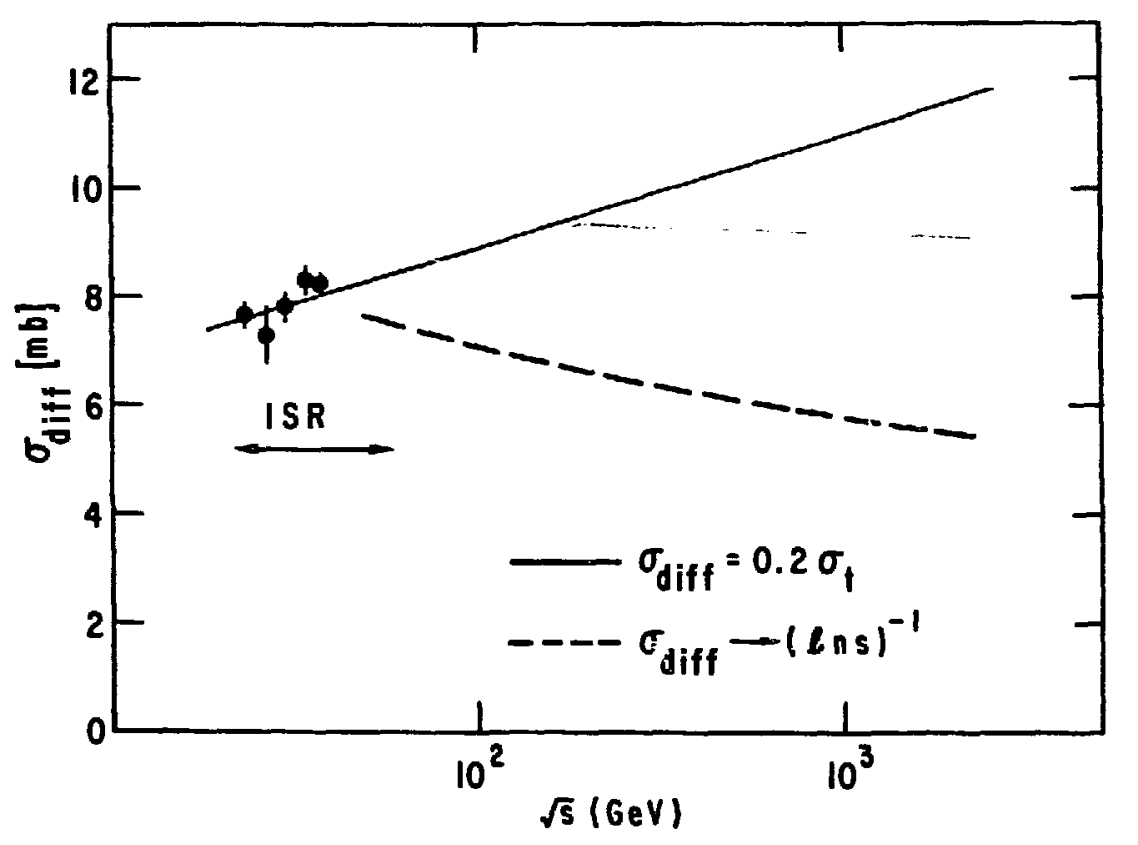

F1g. 2 


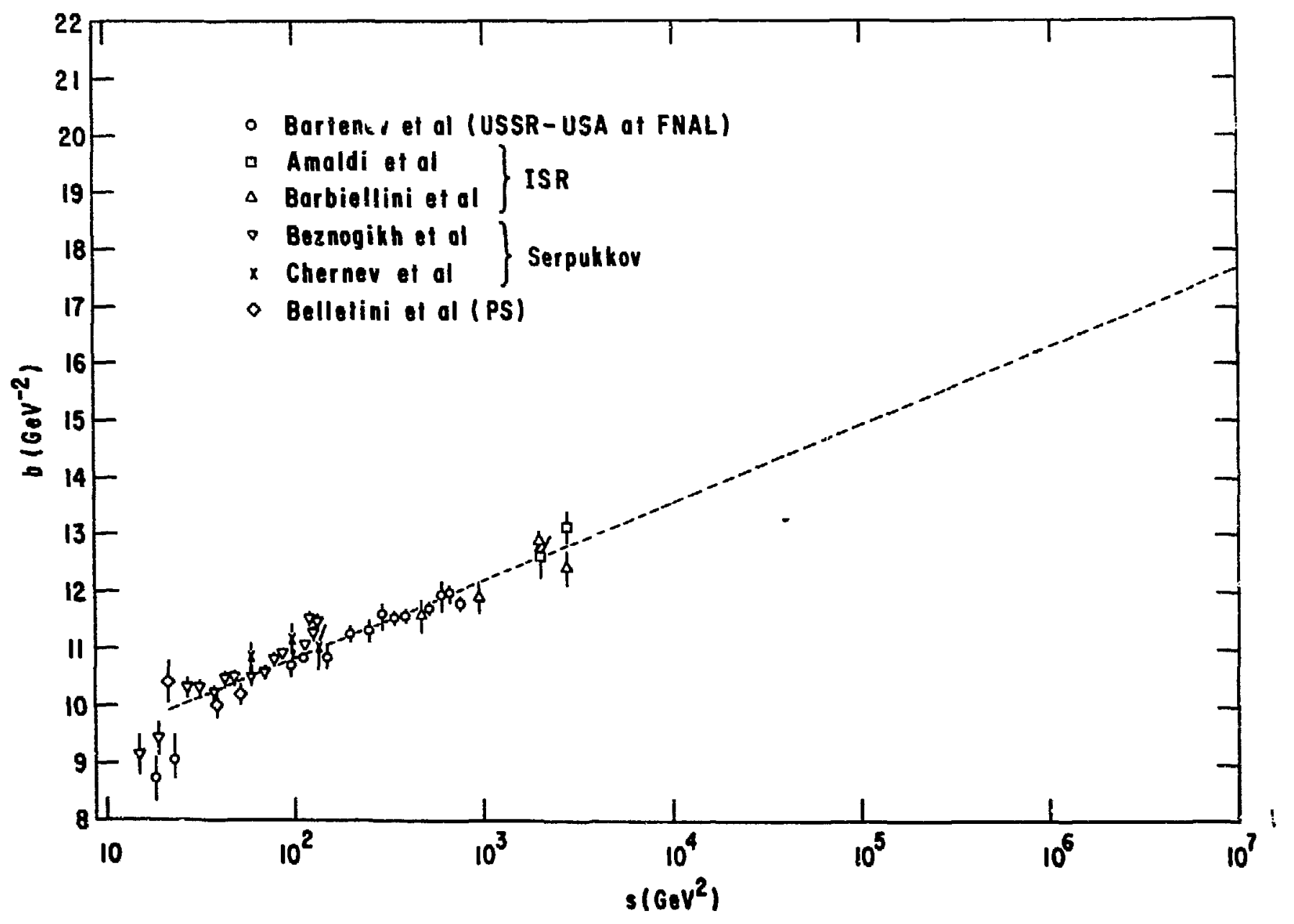

Fig. 3 


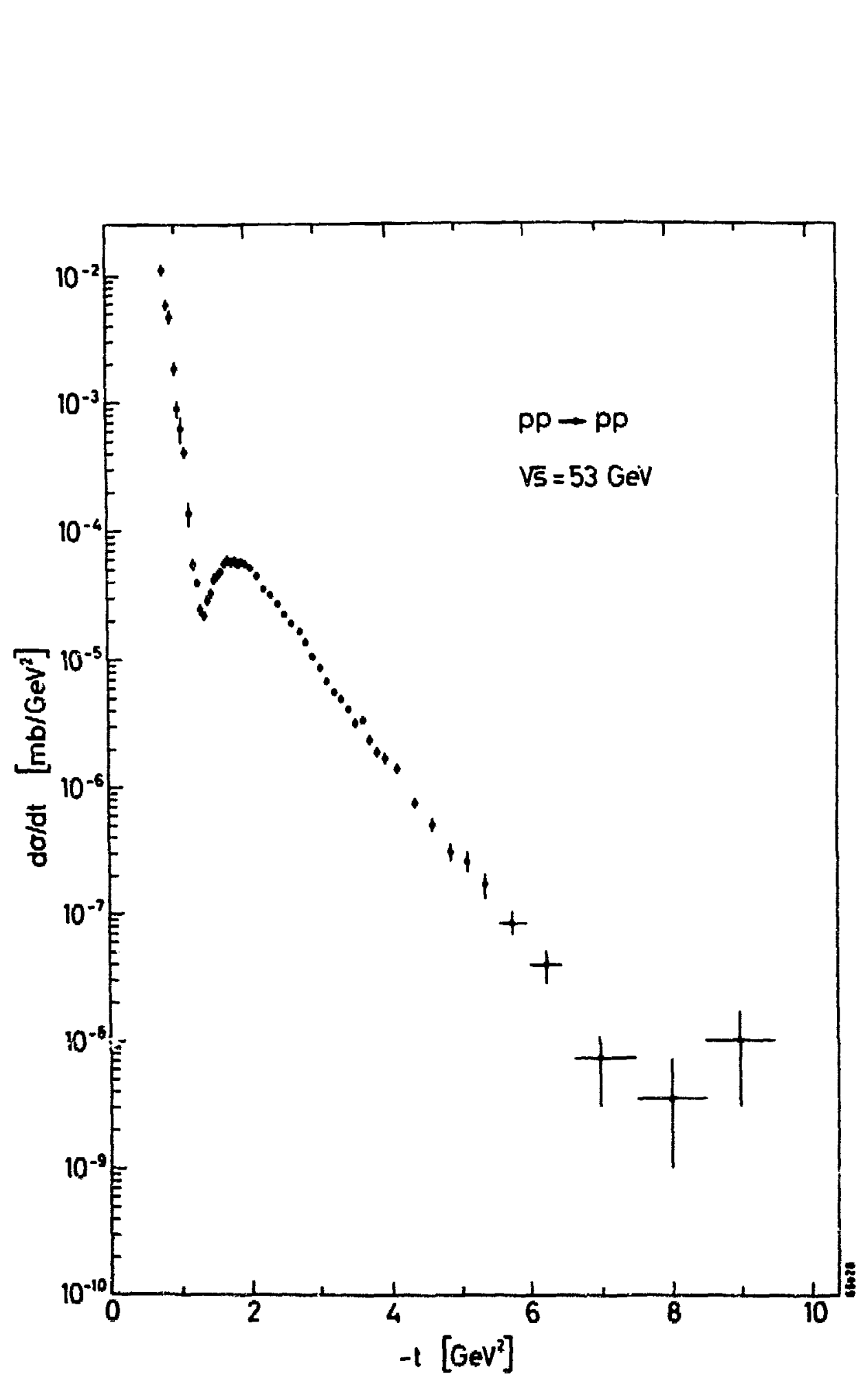

. 


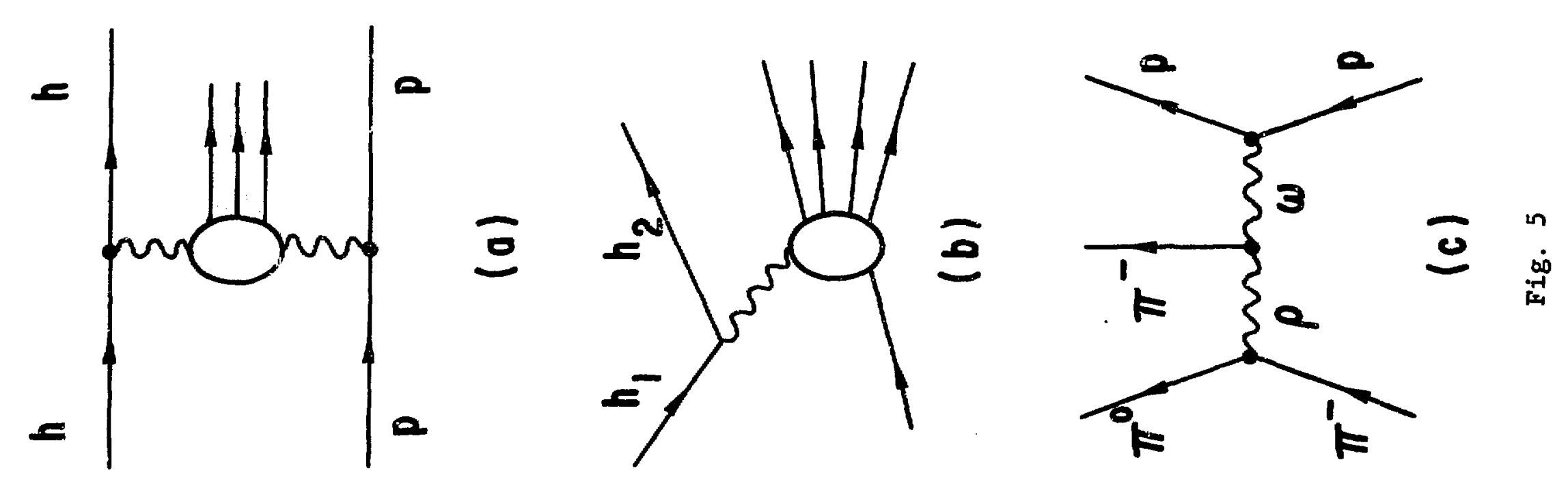




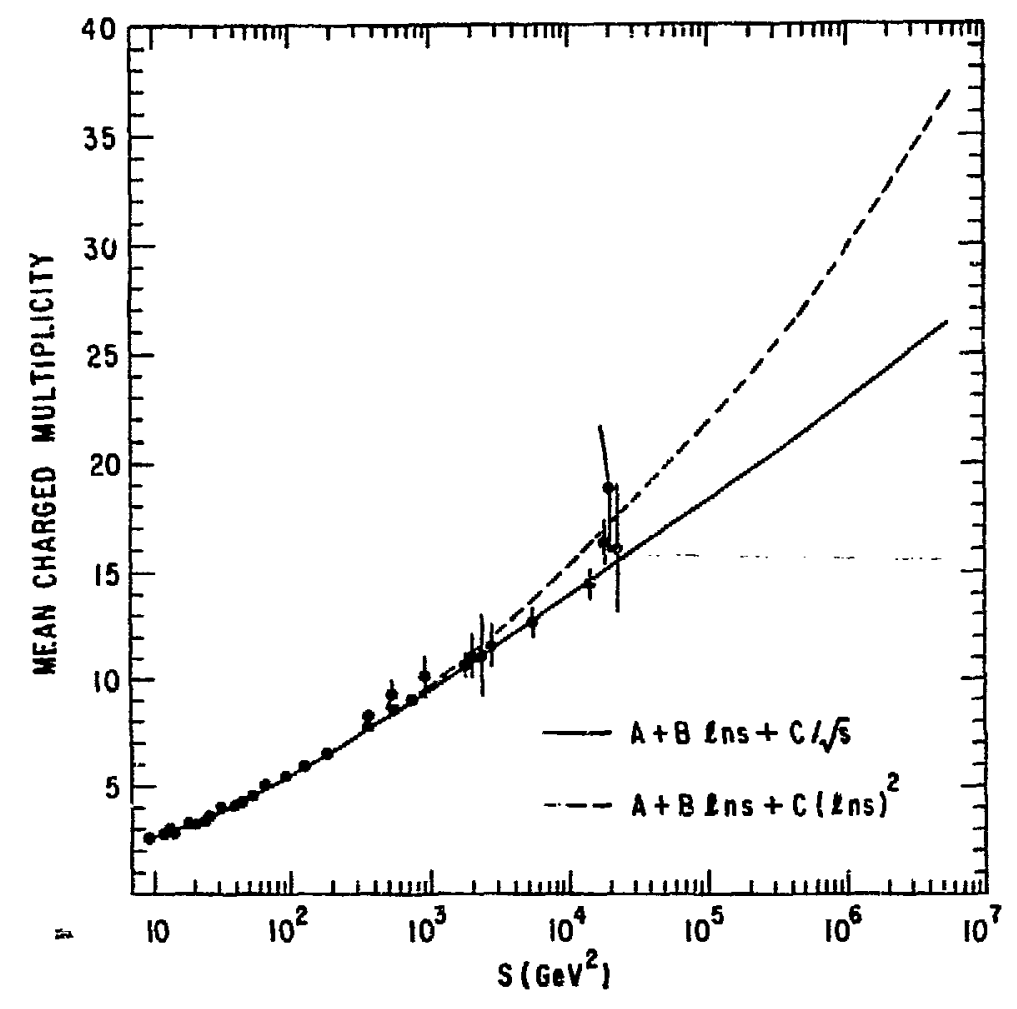

Fig. 6

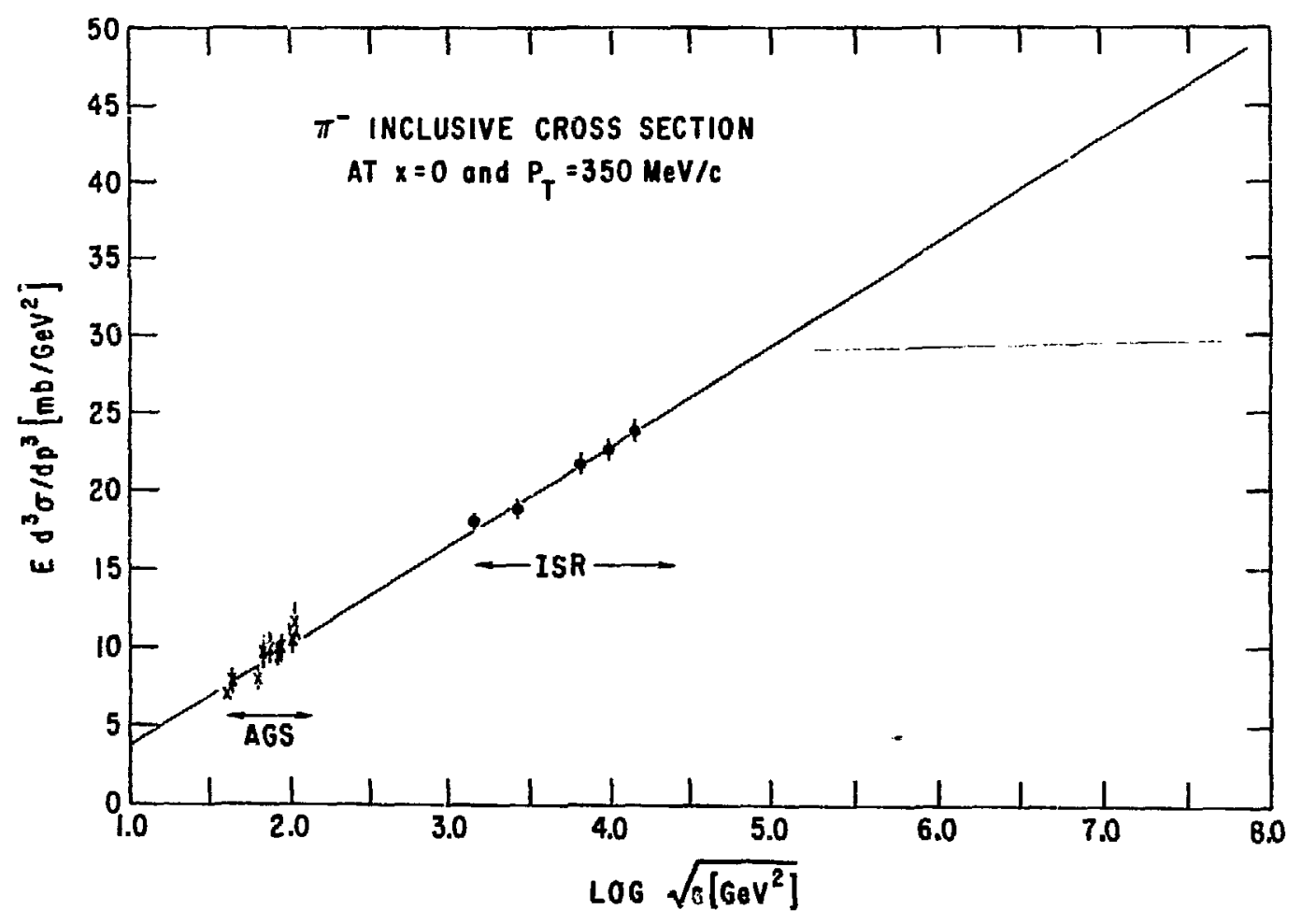

Fig. 7 